\title{
POLARIZED RADIO EMISSION FROM NGC 6946
}

\author{
R. BECK ${ }^{1}$, U.R. BUCZILOWSKI ${ }^{1}$, J.I. HARNETT ${ }^{2}$ \\ ${ }^{1}$ MaX-Planck-Institut für Radioastronomie \\ Auf dem Hügel 69 \\ D-5300 Bonn 1, F.R.G. \\ ${ }^{2}$ University of Sydney \\ N.S.W. 2006, Australia
}

\begin{abstract}
New VLA radio observations of NGC 6946 reveal a complicated structure of the magnetic field with possibly radial changes in the foreground Faraday rotation, the strength of the uniform field and the particular dynamo mode in operation. The uniform field is strongest in the interarm regions, but shows no deducible large-scale structure in the radius range of the optical spiral arms. The high star formation rate in NGC 6946 apparently increases the turbulence of the interstellar magnetic field.
\end{abstract}

\section{Observations and Results}

NGC 6946 is a moderately active starburst galaxy with prominent spiral structure. Alongside the two Effelsberg measurements at $\lambda 2.8 \mathrm{~cm}$ and $\lambda 6.3 \mathrm{~cm}$ (Klein et al., 1982; Harnett et al., 1989) we have undertaken a 12 hour run with the VLA in its $\mathrm{D}$-configuration at $\lambda 18.0 \mathrm{~cm}(1.66 \mathrm{GHz})$

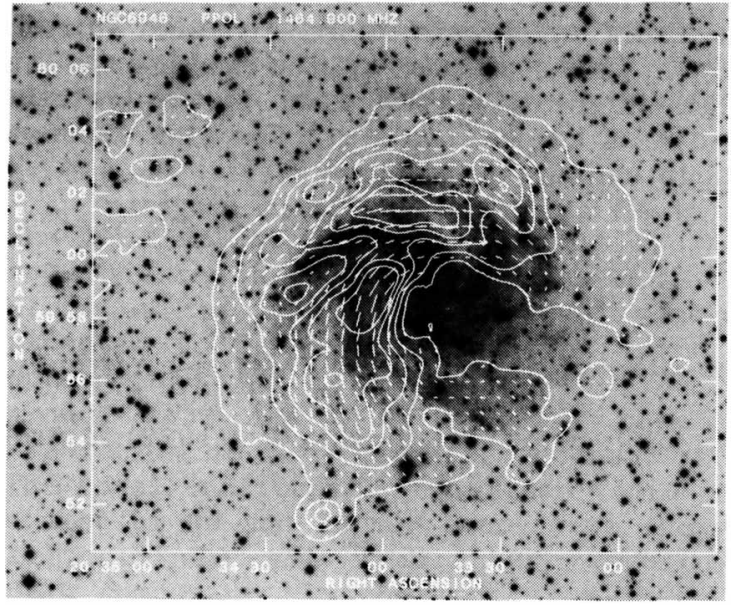

213 and $\lambda 20.5 \mathrm{~cm} \quad(1.46 \mathrm{GHz})$. The synthesized beam of $1^{\prime}$ corresponds to $2.0 \times 2.4$ $\mathrm{kpc}$ in the plane of the galaxy at the assumed distance of $7 \mathrm{Mpc}$.

The Effelsberg observations suggested a bisymmetric magnetic field structure, but allowed no definite conclusions; the field seems to closely follow the optical spiral arms with their largely varying pitch angles (Harnett et al., 1989). The preliminary distribution of the polarized emission at $\lambda 20.5 \mathrm{~cm}$ with polarization vectors is 

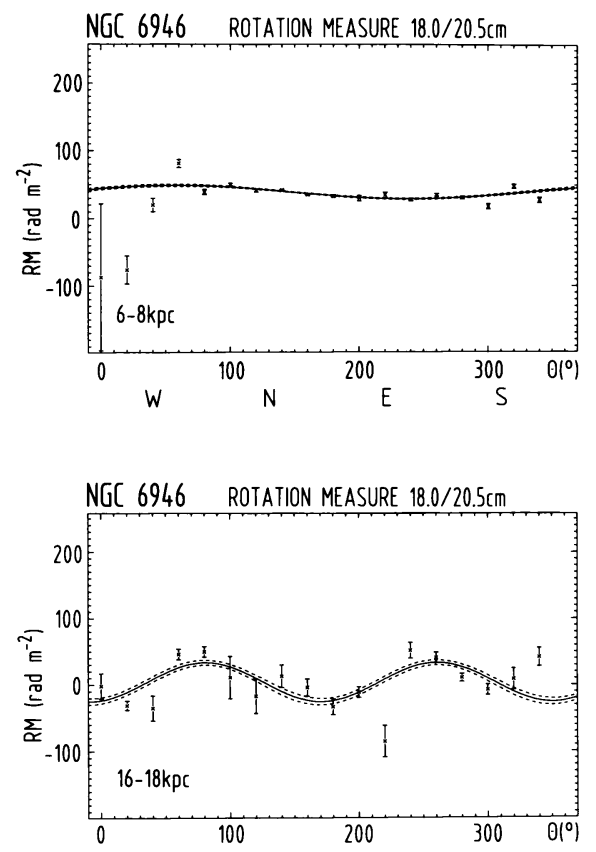

displayed in Fig. 1. The polarized intensity shows an asymmetric distribution as it is high in the northern and eastern parts (both maxima in the interarm region) and very low in the southwestern quadrant where the $\lambda 6.3 \mathrm{~cm}$ Effelsberg map shows a maximum. As the rotation measures are exceptionally large there, the magnetic field probably deviates from the galactic plane and causes strong Faraday depolarization.

We calculated the rotation measures (RM) between $\lambda 18.0$ and $\lambda 20.5 \mathrm{~cm}$ in rings of $2 \mathrm{kpc}$ thickness in the plane of the galaxy (inclination $30^{\circ}, \quad \mathrm{PA}=52^{\circ}$ ) along azimuthal angle $\theta$ (Fig. 2). The ambiguity of the polarization vectors corresponds to $\pm 326 \mathrm{rad} / \mathrm{m}^{2}$ in $\mathrm{RM}$ and may be solved by the $\lambda 6.3 \mathrm{~cm}$ data. Fits indicate a foreground Faraday rotation decreasing with radius. The nearly constant RM between radii of 2 and $\approx 10 \mathrm{kpc}$ allows for a weak uniform field of only $\simeq 2 \mu \mathrm{G}$. However, we see strong polarized emission suggesting frequent field reversals within the disk. The $R M(\theta)$ amplitudes increase from $\simeq 10$ to $20 \mathrm{kpc}$.

Between 6 and $8 \mathrm{kpc}$ (Fig. 2a) as well as 12 and $14 \mathrm{kpc}$ the structure of the ordered field is possibly axisymmetric (single-sinusoidal variation). Indications of bisymmetric modes (double-sinusoidal variation) can be seen between 0 and $2 \mathrm{kpc}$ and are most obvious between 16 and $18 \mathrm{kpc}$ (Fig. 2b) - outside the prominent optical spiral structure. Here the amplitude of the variation would indicate a relatively high internal rotation measure of $\simeq 100 \mathrm{rad} / \mathrm{m}^{2}$. The positions of the maxima in the northwest and southeast are consistent with the ones in our rotation measure curve between $\lambda 6.3$ and $\lambda 2.8 \mathrm{~cm}$ for the $6-12 \mathrm{kpc}$ ring. Between 14 and $16 \mathrm{kpc}$ even a higher mode cannot be excluded.

A multi-frequency analysis of all available data is in progress.

\section{References}

Harnett, J.I., Beck, R. and Buczilowski, U.R. (1989) Astron. Astrophys. 208, 32-38.

Klein, U., Beck, R., Buczilowski, U.R. and Wielebinski, R. (1982) Astron. Astrophys. 108, 176-187. 